\title{
Resveratrol inhibits cell cycle progression by targeting Aurora kinase A and Polo-like kinase 1 in breast cancer cells
}

\author{
RUBICELI MEDINA-AGUILAR ${ }^{1}$, LAURENCE A. MARCHAT ${ }^{2}$, ELENA ARECHAGA OCAMPO ${ }^{3}$, \\ PATRICIO GARIGLIO ${ }^{1}$, JAIME GARCÍA MENA ${ }^{1}$, NICOLÁS VILLEGAS SEPÚLVEDA ${ }^{4}$, \\ MACARIO MARTÍNEZ CASTILLO ${ }^{4}$ and CÉSAR LÓPEZ-CAMARILLO ${ }^{5}$
}

\author{
${ }^{1}$ Department of Genetics and Molecular Biology, CINVESTAV-IPN, Mexico D.F.; ${ }^{2}$ Molecular Biomedicine Program \\ and Biotechnology Network, National School of Medicine and Homeopathy, National Polytechnic Institute, \\ Mexico D.F.; ${ }^{3}$ Natural Sciences Department, Metropolitan Autonomous University, Mexico D.F.; \\ ${ }^{4}$ Department of Molecular Biomedicine, CINVESTAV-IPN, Mexico D.F.; ${ }^{5}$ Oncogenomics \\ and Cancer Proteomics Laboratory, Universidad Autónoma de la Ciudad de México, Mexico D.F., Mexico
}

Received December 4, 2015; Accepted January 8, 2016

DOI: $10.3892 /$ or.2016.4728

\begin{abstract}
The Aurora protein kinase (AURKA) and the Polo-like kinase-1 (PLK1) activate the cell cycle, and they are considered promising druggable targets in cancer therapy. However, resistance to chemotherapy and to specific small-molecule inhibitors is common in cancer patients; thus alternative therapeutic approaches are needed to overcome clinical resistance. Here, we showed that the dietary compound resveratrol suppressed the cell cycle by targeting AURKA and PLK1 kinases. First, we identified genes modulated by resveratrol using a genome-wide analysis of gene expression in MDA-MB-231 breast cancer cells. Transcriptional profiling indicated that 375 genes were modulated at $24 \mathrm{~h}$ after resveratrol intervention, whereas 579 genes were regulated at $48 \mathrm{~h}$. Of these, 290 genes were deregulated in common at 24 and $48 \mathrm{~h}$. Interestingly, a significant decrease in the expression of genes involved in the cell cycle, DNA repair, cytoskeleton organization, and angiogenesis was detected. In particular, AURKA and PLK1 kinases were downregulated by resveratrol at $24 \mathrm{~h}$. In addition the BRCA1 gene, an AURKA/PLK1 inhibitor, was upregulated at $24 \mathrm{~h}$ of treatment. Moreover, two wellknown resveratrol effectors, cyclin D1 (CCND1) and cyclin B1 (CCNB1), were also repressed at both times. Congruently, we found that resveratrol impaired G1/S phase transition in both
\end{abstract}

Correspondence to: Dr César López-Camarillo, Oncogenomics and Cancer Proteomics Laboratory, Universidad Autónoma de la Ciudad de México, San Lorenzo 290, Col. Del Valle, CP 03100, Mexico D.F., Mexico

E-mail: genomicas@yahoo.com.mx

Abbreviations: AURKA, Aurora protein kinase; PLK1, Polo-like kinase-1; CCND1, cyclin D1; CCNB1, cyclin B1

Key words: breast cancer, resveratrol, transcriptome, cell cycle, AURKA, PLK1, BRCA1
MDA-MB-231 and MCF-7 cells. By western blot assays, we confirmed that resveratrol suppressed AURKA, CCND1 and CCNB1 at 24 and $48 \mathrm{~h}$. In summary, we showed for the first time that resveratrol regulates cell cycle progression by targeting AURKA and PLK1. Our findings highlight the potential use of resveratrol as an adjuvant therapy for breast cancer.

\section{Introduction}

Cancer development results from the interaction between genetic factors, environment and dietary factors (1). Breast cancer accounts for 522,000 deaths and is the most frequently diagnosed cancer among women, with 1.7 million cases worldwide in 2012 (2). Even though new strategies for breast cancer therapy are being developed, unfortunately cancer frequently recurs in patients after first-line treatment. Tumor progression is commonly associated with disorders in the regulation of the cell cycle. The Aurora kinase A (AURKA) and Polo-like kinases 1 (PLK1) proteins play key roles in cell cycle phase transition as they co-regulate multiple processes such as mitotic spindle formation, sister chromatid resolution, chromosome spindle connections and cytokinesis. Moreover, AURKA and breast cancer 1 susceptibility (BRCA1) proteins may interplay to control the cell cycle, chromosome polyploidy, and tumorigenesis. The silencing of AURKA was found to suppress cell cycle progression mainly by blocking G1-S and G2-M transition, while the disruption of BRCA1 was found to promote cell cycle progression through induction of G1-S and G2-M transition (3). On the other hand, targeted inhibition of PLK1 can produce antitumor effects, while its constitutive expression in mammalian cells leads to malignant transformation suggesting that aberrant PLK1 expression is oncogenic (4). Because of its important role in cancer, diverse pharmacologic inhibitors have been developed for AURKA (MLN8054, ENMD-2076, Hesperidin and VX680) and PLK1 (BI2536, GSK-461364, Poloxin, CYC-800 and HMN-241) proteins (5). However, resistance to these developed inhibitory molecules is frequently observed in clinical practice; thus 
alternative therapeutic approaches are needed to overcome resistance and improve patient survival and outcome.

The use of phytochemicals from vegetables, fruits, spices, teas, herbs and medicinal plants, represents one of the most feasible means of cancer chemoprevention (6). Resveratrol (3,5,4'-trihydroxy-trans-stilbene) is a phytoalexin found in grapes, berries, peanuts, chocolate, red wine, herbs and plants, and its concentration increases during environmental stress and pathogen invasion. Notably, this natural polyphenol possesses cancer chemopreventive and chemotherapeutic activites by inhibiting diverse cellular events associated with tumor development (7). In fact, resveratrol represents a promising class of anticancer drug, since it acts by targeting multiple proteins in cancer cells having limited toxic effects on normal cells. It also has a potential antitumor effect against the progression of various types of cancer such as prostate, breast, liver, skin and lung cancer (8). Resveratrol exhibits antitumor activity in breast cancer through suppression of migration, invasion and cell cycle progression. It also activates apoptosis leading to chemosensitization of tumor cells in vitro (9). Importantly, resveratrol triggers numerous intracellular pathways leading to cell growth and cell cycle arrest (10). In this study, we evaluated the changes in gene expression profiles induced by resveratrol treatment of MDA-MB-231 breast cancer cells to identify modulated genes that may represent potential therapeutic targets. Interestingly, we found that resveratrol regulates cell cycle progression by targeting AURKA, PLK1 and BRCA1. Our data highlight the potential use of resveratrol in combination with antineoplastic drugs as adjuvant therapy in breast cancer.

\section{Materials and methods}

Cell cultures. MCF-7 and MDA-MB-231 breast cancer cell lines were obtained from the American Type Culture Collection (ATCC; Manassas, VA, USA). Cells were maintained in Dulbecco's modified Eagle's minimal essential medium (DMEM), supplemented with $10 \%$ fetal bovine serum and antibiotics $(100 \mathrm{U} / \mathrm{ml}$ penicillin and $100 \mathrm{U} / \mathrm{ml}$ streptomycin) at $37^{\circ} \mathrm{C}$ in a humidified atmosphere of $5 \% \mathrm{CO}_{2}$.

Reagents. Resveratrol was purchased from Sigma-Aldrich (St. Louis, MO, USA), and dissolved at a concentration of $80 \mathrm{mmol} / \mathrm{l}$ in ethanol, stored at $-20^{\circ} \mathrm{C}$ and diluted with DMEM to a $100-\mu \mathrm{M}$ working concentration.

DNA microarrays and validation. Total RNA was processed and hybridized into the GeneChip Human Gene 1.0 ST (Affymetrix Inc., Santa Clara, CA, USA), following the manufacturer's recommendations. Microarray quality assessment, condensing of the probe sets, data normalization, and filtering were conducted using the Transcriptome Analysis Console version 2.0 (Affymetrix). To identify those biological processes that show differentially expressed genes, we used KEGG and the Database for Annotation, Visualization and Integrated Discovery (DAVID) v6.7 for functional annotation. To validate the microarray data, real-time quantitative PCR (RT-qPCR) was performed for 13 selected genes using specific primers (Table I). The SYBR-Green reaction was carried out using a QuantiTect SYBR Green PCR reagents kit (Qiagen) following the manufacturer's recommendations. GADPH expression was used as control. Samples were tested in triplicate and data were analyzed using the $2^{-\Delta \Delta C t}$ method (11).

Cell cycle analysis. MDA-MB-231 and MCF-7 cells were plated at a density of $5 \times 10^{4}$ cells $/ \mathrm{ml}$ for $24 \mathrm{~h}$, and then treated with resveratrol $(100 \mu \mathrm{M})$ for 24 and $48 \mathrm{~h}$. Cells were harvested by trypsinization and fixed in ice-cold $70 \%$ methanol overnight at $-20^{\circ} \mathrm{C}$. Cells were centrifuged at $1,200 \mathrm{rpm}$ for $5 \mathrm{~min}$ and incubated with a propidium iodide (PI) working solution $\left(100 \mu \mathrm{g} / \mathrm{ml} \mathrm{PI}\right.$ and $100 \mu \mathrm{g} / \mathrm{ml}$ RNase A) for $30 \mathrm{~min}$ at $37^{\circ} \mathrm{C}$. Cell cycle distribution was analyzed using a FACScan flow cytometer (FACSCalibur; Becton-Dickinson).

Western blotting. Protein extracts $(50 \mu \mathrm{g})$ were separated by $12 \%$ SDS-PAGE and electrotransferred to a nitrocellulose membrane (Bio-Rad). Membranes were blocked with 5\% nonfat milk in PBS with $0.1 \%$ Tween-20 and then probed with primary antibodies as follows: anti-AURKA $(1: 1,000$; Cell Signaling Technology), anti-PLK1 (1:500), anti-cyclin D1 $(1: 1,000)$, anti-cyclin B1 $(1: 1,000)$ and anti-BRCA1 $(1: 1,000)$ (all from Santa Cruz Biotechnology), followed by secondary antibodies, anti-rabbit $(1: 2,500)$ or anti-mouse $(1: 2,500)$ (both from Santa Cruz Biotechnology). The membranes were probed with anti- $\alpha$-tubulin (1:1,000; Sigma-Aldrich) or actin $(1: 1,000 ;$ Abcam $)$ as a loading control. Immunoreactive bands were developed using the ECL chemiluminescence system (Amersham Pharmacia Biotech).

Statistical analysis. A two-way ANOVA was performed to identify differentially expressed genes of the microarray data. Only genes with statistically significant differences in expression levels ( $\mathrm{P}$-value $<0.05$ ) and a fold change criteria of $\geq 1.5$ were included in the final set of differentially expressed genes. RT-qPCR results were analyzed using the Student's test. Differences of $\mathrm{P}<0.05$ were considered statistically significant. Values are presented as the mean \pm standard deviation of three independent experiments.

\section{Results}

Resveratrol induces changes in the transcriptome of human $M D A-M B-231$ breast cancer cells. Resveratrol is able to inhibit cell viability through apoptosis activation in diverse types of cancer cells $(12,13)$. Here, the effects of resveratrol $(100 \mu \mathrm{M})$ on cell viability and apoptosis of triple-negative MDA-MB-231 breast cancer cells were evaluated. Results of the MTT, Annexin V and TUNEL assays confirmed that resveratrol treatment was able to significantly $(\mathrm{P}<0.05)$ inhibit cell viability and induce apoptosis after 24 and 48 h treatments (data not shown). These results concur with previous reports on diverse types of cancer cells (14-17). Then, in order to obtain insight concerning the cellular transcripts modulated by resveratrol that may explain its anticancer effects, we performed a genome-wide analysis of the transcriptome of MDA-MB-231 breast cancer cells after treatment with resveratrol $(100 \mu \mathrm{M})$ for 24 and $48 \mathrm{~h}$ using DNA microarrays. Data from two biological replicates were analyzed, normalized, and raw P-values adjusted. Only genes with a significant fold change $(\mathrm{FC}>1.5 ; \mathrm{P}<0.05)$ were included in this analysis. Transcriptional profiling showed that 375 genes (206 upregu- 
Table I. Primer sequences for quantitative reverse-transcription polymerase chain reaction.

\begin{tabular}{llll}
\hline Gene & \multicolumn{1}{c}{ Forward primer 5'-3' } & \multicolumn{1}{c}{ Reverse primer 5'-3' } & Reference sequence \\
\hline AURKA & CGTGTTCTCGTGACTCAGCA & TGGTTGCCTGCAATTGCTTC & NM_003600 \\
FUT11 & CAACGGCTTCGAGTGTTTCG & CTCTTTCCAACTGTCATTCTCAGG & NM_173540 \\
HK2 & TCCAACCTTAGGCTTGCCATT & CTTGGACATGGGATGGGGTG & NM_000189 \\
UBASH3B & AAATTCCCGGACTGCTAGGC & CTCTGACGCTTCCTGGTGAG & NM_032873 \\
CPA4 & CCTGCAGGCCCTTTTAGACA & CGGCCGGTTTCAAACGAAT & NM_016352 \\
DSCC1 & CAAGCTGTGCTGTGCAGTAA & TGGGTCTACGTCTTCTTAATCCC & NM_024094 \\
NEIL3 & TGGAAGTGCAGCTCACCAAA & AGCACATCACCTAGCATCCG & NM_018248 \\
PEG10 & GAGAACAGCGGAGAAGGTCC & TCAAAACCCGCTTATTTCGCA & NM_001172437 \\
DDR2 & CCTACAAGTTGCCTGGGGTT & AGGCTACAGTCTCCCTCTGG & NM_001014796 \\
MSH2 & GGAGGTGAGGAGGTTTCGAC & GTATAGAAGTCGCCCCGGTC & NM_000251 \\
UGT2B11 & TGCCAAGATCCCACAAAAGGT & TGGAATGCCCACCATAGGGA & NM_001073 \\
CYP1B1 & AGTTCTTGAGGCACTGCGAA & GTGATAGTGGCCGGTACGTT & NM_000104 \\
BRCA1 & ACGGAAAAGCGCGGGAATTA & TCTTCAACGCGAAGAGCAGA & NM_007294 \\
GAPDH & CCCCACCACACTGAATCTCC & GTACATGACAAGGTGCGGCT & NM_001289746.1 \\
\hline
\end{tabular}

A
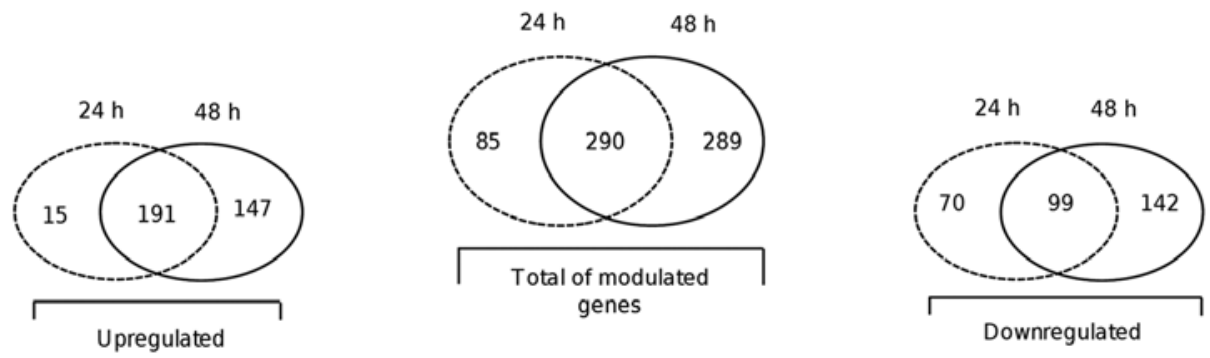

B
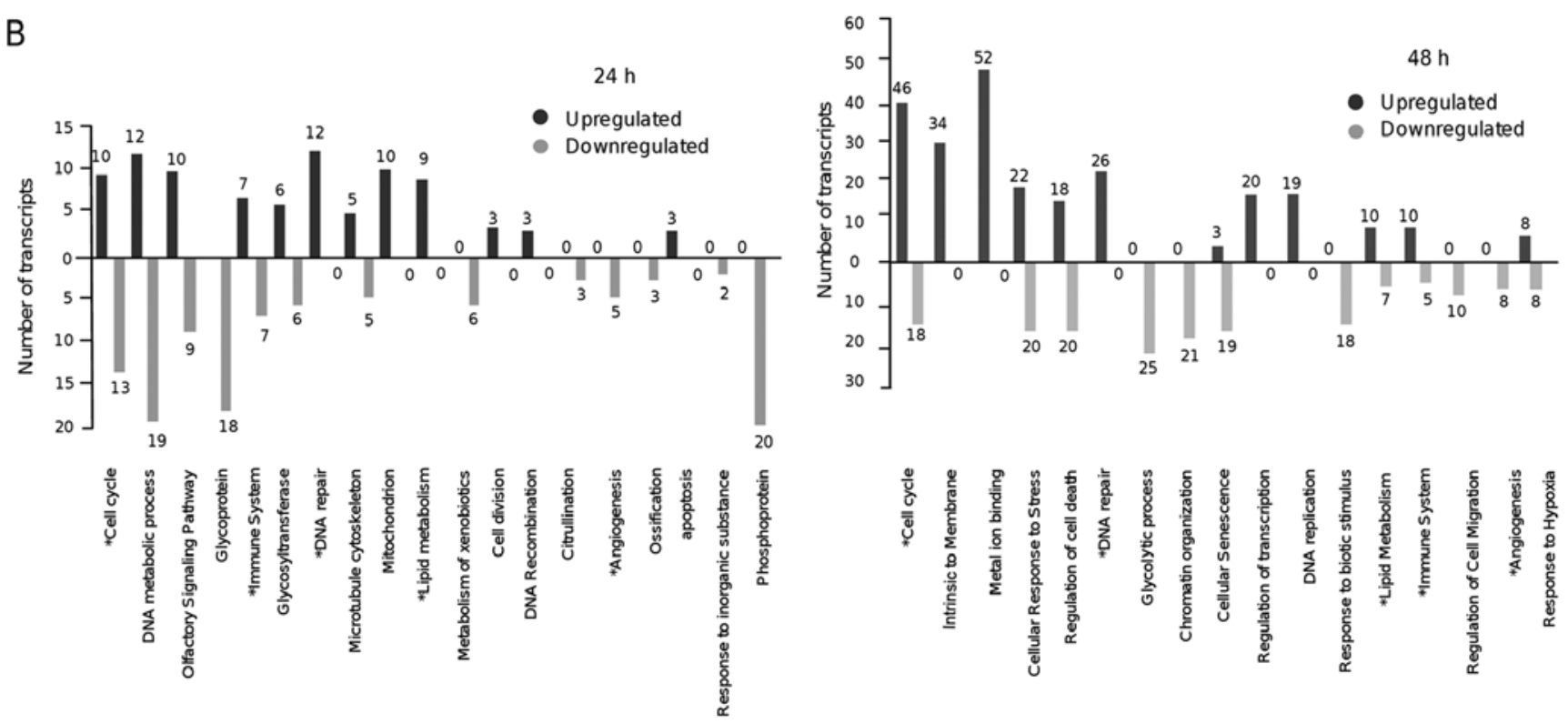

Figure 1. (A) Venn diagrams of genes regulated after resveratrol treatment in MDA-MB-231 breast cancer cells. Overlap of the modulated genes after resveratrol treatment $(100 \mu \mathrm{M})$. Dotted and solid lines indicate genes regulated at 24 and $48 \mathrm{~h}$ after treatment, respectively. Intersected areas of the circles indicate the number of common genes modulated at 24 and $48 \mathrm{~h}$. (B) Distribution of modulated genes into functional categories.

lated and 169 downregulated) were significantly modulated at $24 \mathrm{~h}$ (Table II). After a 48-h treatment with resveratrol, changes in the expression of 579 genes were detected (338 upregulated and 241 downregulated). Of these, 290 genes (30\%) were modulated in common at 24 and 48 h (Fig. 1A).
Overview of the modulated genes by resveratrol in MDA-MB231 breast cancer cells. To evaluate the impact of resveratrol on cellular processes and pathways of MDA-MB-231 cells, a bioinformatic analysis of the DNA microarray data set was performed. We used the Database for Annotation, Visualization 
Table II. Deregulated genes and cellular processes modulated by resveratrol treatment at $24 \mathrm{~h}$.

\begin{tabular}{|c|c|c|}
\hline Process & $\begin{array}{l}\text { No. of } \\
\text { genes }\end{array}$ & Gene name \\
\hline Cell cycle & 22 & $\begin{array}{l}\text { TXNIP, CCNB1, FAM83D, CDCA8, HJURP, PLK1, CENPA, DLGAP5, BUB1, AURKA, ASPM, } \\
\text { WEE1, CDC6, DSN1, E2F8, MIS12, SESN3, CCNE2, CCNE1, PSMC3IP, MNS1, DSCC1 }\end{array}$ \\
\hline DNA metabolic process & 31 & $\begin{array}{l}\text { HIST1H2AB, H1F0, HIST1H2BB, HIST1H3J, HIST1H2AE, HIST1H2BM, H2BFS, } \\
\text { HIST1H2BK, HIST1H3A, HIST1H3B, HIST1H2AH, HIST1H3C, HIST1H3D, HIST1H2AJ, } \\
\text { HIST1H3E, HIST1H3F, HIST1H3G, HIST1H3H, HIST1H3I, CLSPN, CDC6, GINS2, DNAH14, } \\
\text { FAM175A, DTL, GINS3, RNASEH2A, MCM6, CCNE2, FANCL, PRIM1 }\end{array}$ \\
\hline $\begin{array}{l}\text { Olfactory signaling } \\
\text { pathway }\end{array}$ & 19 & $\begin{array}{l}\text { OR13C4, OR5P3, OR5P2, ARHGAP11A, ARHGAP11B, SLC6A6, SLC5A3, SLC14A1, } \\
\text { SLC2A3, HK2, C5, SAA2, CCL20, PRICKLE1, KITLG, DKK1, DKK1, NAPEPLD, MAP2 }\end{array}$ \\
\hline Glycoprotein & 18 & $\begin{array}{l}\text { SLC5A3, NOG, TNC, IGHM, OR13C4, SLC2A3, CLEC2B, IGHA1, OR5P3, OR5P2, IL24, } \\
\text { IGHV3-11, EYS, GPR110, SLC6A6, STC1, TM4SF1, SLC14A1 }\end{array}$ \\
\hline Immune system & 14 & $\begin{array}{l}\text { IGHG1, IGHV311, IGV330, IGV348, SCFV, IGHA1, IGHM, C5, IL18, ICAM2, TRIM68, IL18, } \\
\text { CLU, C5 }\end{array}$ \\
\hline Glycosyltransferase & 12 & $\begin{array}{l}\text { ALOX5AP, GPSM2, ARHGAP11B, ARHGAP11A, DEPDC1, ERRFI1, UNG, AMY1C, AMY2A, } \\
\text { AMY1B, AMY1A, GBA }\end{array}$ \\
\hline DNA repair & 12 & BRIP1, UNG, BRCA2, MLH1, FANCL, BRCA1, EXO1, CLSPN, BLM, ESCO2, DNA2, POLN \\
\hline $\begin{array}{l}\text { Microtubule } \\
\text { cytoskeleton }\end{array}$ & 10 & CCNB1, FAM83D, KIF14, CDCA8, KIF20A, CDC6, ROCK1P1, DNAH14, MAP2, DNAH6 \\
\hline Mitochondrion & 10 & UNG, KIAA0101, ASAH2, SLIT3, TIMM8A, TST, ASAH2C, ASAH2B, SLC25A19, SLC27A2 \\
\hline Lipid metabolism & 9 & ACADSB, NAPEPLD, LIPK, ASAH2C, ASAH2B, SLC27A2, ASAH2, GBA, AADAC \\
\hline $\begin{array}{l}\text { Metabolism of enobiotics } \\
\text { by cytochrome P } 450\end{array}$ & 6 & UGT2B17, CYP1B1, UGT2B11, UGT2B10, UGT2B15, UGT2B7 \\
\hline Cell division & 3 & CDC6, DSN1, MIS12 \\
\hline DNA recombination & 3 & EXO1, BLM, PSMC3IP \\
\hline Citrullination & 3 & HIST1H2AH, HIST1H3D, HIST1H3E \\
\hline Angiogenesis & 5 & JUN, VEGFA, LOX, ANGPTL4, PTGS2 \\
\hline Ossification & 3 & NOG, STC1, RUNX2 \\
\hline $\begin{array}{l}\text { Induction of apoptosis } \\
\text { by intracellular signals }\end{array}$ & 3 & TP53I3, CCNE1, SESN3 \\
\hline $\begin{array}{l}\text { Response to inorganic } \\
\text { substance }\end{array}$ & 2 & TXNIP, NDRG1 \\
\hline Phosphoprotein & 20 & $\begin{array}{l}\text { PRR11, CDCA8, H2BFS, HIST1H2BK, ANKZF1, NFIL3, ERRFI1, ASPM, KIF14, ND2, } \\
\text { TMEM71, DEPDC1, SLITRK6, PFKFB3, GNE, TNC, LPXN, GPSM2, NDRG1, BHLHE40 }\end{array}$ \\
\hline
\end{tabular}

Suppressed genes are denoted in bold print. Upregulated genes are denoted in normal print.

and Integrated Discovery (DAVID) and Reactome Database for functional annotation and visualization of the expression data in the KEGG biological pathway context. Gene ontology category analysis indicated that a number of genes involved in cancer-related processes were modulated by resveratrol at both times (Fig. 1B). Of these, 47 suppressed genes are well-known oncogenes, which highlight the potential effects of resveratrol on the suppression of cancer-related processes. Notably, a significant decrease in the expression of genes involved in the cell cycle, DNA metabolic processes, cytoskeleton organization, metabolism of xenobiotics by cytochrome P450, and angiogenesis was detected at $24 \mathrm{~h}$ after resveratrol treat- ment (Table II). Several of these genes, including SLC6A6, HK2, GPR110, IGHG1, ALOX5AP, CCNB1, CYP1B1, JUN, VEGFA, ANGPTL4 and histones, are clinical therapeutic targets in several types of cancers (18-20). Remarkably, after a 24-h resveratrol treatment we identified the overexpression of a set of DNA repair genes including BRIP1, UNG, BRCA2, MLH1, FANCL, BRCA1, EXO1, CLSPN, BLM, ESCO2, DNA2, POLN (Table II).

In contrast, at $48 \mathrm{~h}$ after resveratrol treatment we detected the upregulation of genes involved in cellular response to stress, regulation of cell death, glycolytic process, chromatin organization and cellular senescence. Notably, DNA 
Table III. Deregulated genes and cellular processes modulated by resveratrol treatment at $48 \mathrm{~h}$.

\begin{tabular}{lll}
\hline & No. of \\
genes & Gene name \\
\hline
\end{tabular}

Cell cycle

Intrinsic to membrane

Metal ion binding

Cellular response to stress

Regulation of cell death

DNA repair

Glycolytic

process

Chromatin

organization

Senescence

Regulation of transcription

DNA replication

Response to biotic stimulus

Metabolism of lipids and lipoproteins

Immune system

Regulation of cell migration

Angiogenesis

Response to hypoxia
64 AURKA, PLK1, HIST1H3F, HIST1H2AJ, JUN, ARHGAP18, KIF20A, SH3KBP, EDN1, HIST1H2BH, HIST1H2AH, HIST1H1E, HIST1H2BM, H1FO, HIST1H1C, H2BFS, HIST1H2BB, PSMD11, MSH2, ARL3, DSN1, E2F1, E2F8, FBXO43, HAUS4, MPHOSPH9, MTBP, PSMC31B, TSPYL2, AVPI, CASP8AP2, CDC6, CDT1, CLSPN, CCN1, CCN2, ESCO2, EXO1, MNS1, MND1, MLH1, RBBP8, SESN3, TIMELESS, DSNI, MASTL, CDT1, BLM, PRIM1, PRKACA, CENP, CENP1, RRM2, MLF1IP, RFC5, RFC4, TUBB4B, PSMC3IP, DNA2, CDC6, EMD, TPH1, ALDH7A1, ACADSB

34 MFGE8, MOXD1, OR5L1, PIGX, PLCE1, PSTP1P2, P4HB, RTN3, STAM2, SVIP, SLC16A12, SLC27A2, SLC35AS, SLC38A4, SLC40A1, SLC5A10, ATRNL1, B3GAT3, CLGN, C14orf21, DCXR, ENTPD3, EMD, EDNRB, EPHXI, GBA, GBP2, TMEM232, ITGB3, MGAT4A, TSPANI2, TSPANI3, TMEMIO6C, UCP2

51 PEG10, CPA4, ARL3, ATP8A1, EFCAB5, EFEMP1, FBX043, FANCL, GPR98, NAPEPLD, RFESD, KDMIB, AMY1A, AMY1C, AMY1B, AMY2A, AMY2B, BIRC3, B3GAT3, CAPS2, CLGN, C4orf21, ENTPD3, EME1, FBNI, GUCYIB3, IDH2, MATN2, MOXD1, PLCE1, PRICKLE1, SLC38A4, SLC40A1, SLC5A10, TCF19, TRIM68, TPH1, UBR7, VSNL1, ZC3H6, ZNF367, ZNF443, ZNF594, ZNF665, ZNF678, ZNF682, ZNF701, ZNF724P, ZNF730, ZNF799, ZNF845

42 ALOX5AP, PTGS2, CYP1B1, PLA2G4A, SLC2A3, TNC, LDHA, KIF204, PSMD11, H2BFS, SH3KBP1, APOBEC3G, BHLHL40, UGT2B10, UGT2B11, UGT2B15, UGT2B17, CYP1B1, GPI, HK2, BRIP1, ATAD5, FAM175A, CCL20, CLU, C5, CFB, ITGB3, PTX3, SAA2, SAA4, TFPI2, DCXR, TP53I3, VNN2, EPHXI, GGH, MFGE8, SLIT3, RPS6KA5, P4HB, FBN1

38 HIST1H1E, PSMD11, H1F0, HIST1H1C, TNFRSF10D, BNIP3, MMP9, PHLDA1, SERPINB2, TNFRSF10D, IL24, DDIT4, NOG, EDN1, OR5P2, OR5P3, ALG3, PSMD11, SLC2A3, TMEM87B, PSMD11, H1F0, HIST1H1C, TNFRSF10D, BNIP3, PHLDA1, SERPINB2, TNFRSF10D, IL24, DDIT4, NOG, EDN1, OR5P2, OR5P3, ALG3, PSMD11, SLC2A3, TMEM87B

26 NEIL3, BRIPI, BLM, DNA2, FANCB, FANCD2, FANCL, RAD54L, RECQL, BRCA1, BRCA2, CLSPN, EME1, ESCO2, EXO1, FAM175A, MLH1, MSH2, POLN, RFC5, RBBP8, UNG, MNS1, PIGX, MGAT4A, ST6GALNA

25 PFKB3, TPI1P1, GPI, LDHA, PGK1, ND2, FUT11, WDR74, BHLHB9, DDX21, MLKL, ANK2F1, ERRFI1, CHORDC1, LTV1, TM45F19, PFKFB3, TKTL2, LPXN, RAB43, TMEM87B, NDRG1, HSPA8, RPS24

22 PLK1, RARA, NEDD4, VARS, HIST1H2BB, RPS24, PSMD11, HIST1H2BM, RUNX2, H2BFS, HIST1H3F, HIST1H2AJ, HIST1H2BH, HIST1H2AH, HIST1H1E, HIST1H2BM, ALG3, PLA2G4A, HK2, NEDD4, RPS24, HAS2

22 HIST1H3F, JUN, HIST1H2AJ, HIST1H2BH, HIST1H2AH, HIST1H1E, HIST1H2BM, H1F0, HIST1H1C, H2BFS, PSMD11, APOBC3G, SLC2A3, VEGFA, PLA2G4A, EDN1, MMP3, BP1, HAS1, E2F1, CCNE1, CCNE2

20 NRIPI, CBX5, CREG1, CDCA2L, TSPYL2, PSMC3IP, MLF1IP, E2F8, ATAD2, PER3, TCF19, ZNF845, ZNF799, ZNF367, ZNF443, ZBF594, ZNF678, ZNF682, ZNF701, ZNF730

19 DSCC1, CDT1, CCDC111, POLN, RNASEH2A, TK1, KIF24, FANCD2, BLM, RFC4, RFC5, RRM2, KITLF, DNA2, PRIM1, TSPYL2, CSF2, ATAD2, ATAD5

18 SLC5A3, SLC14A1, NEDD4, SLC2A3, HK2, HMGCS1, HLADRA, IFITM3, ORM1, RUNX2, NDRG1, BHLHE40, IGLON5, VWC2L, SLITRK6, PLK1, PLOD2, SORD2

17 HMGCS1, ARSJ, GPR110, ERRF11, SLC16A6, MT1F, MTIJP, TSPYL2, MNS1, MGAT4A, PIGX, SLC27A2, GBA, P4HB, ELOVL7, UBR7, FBX016

15 KIF20A, PSMD11, SH3KBP1, IFITM3, EDD4, IL18, GBP2, CFB, CSF2, SAMHD1, BIRC3, TUBB4B, RPS6KA5, CD226, TRIM68

12 NEXN, PLOD2, ARSJ, PTGS2, TNC, SH3KBP1, ARHGAP18, ORM1, S100A11, GPR110

8 ANGPTL4, EDN1, GPI, JUN, ROBO4, VEGFA, LOX, PTGS2

8 SHISA3, DDIT4, PLOD2, VARS, LDHA, RARA, KIF20A, SLC6A6 
A

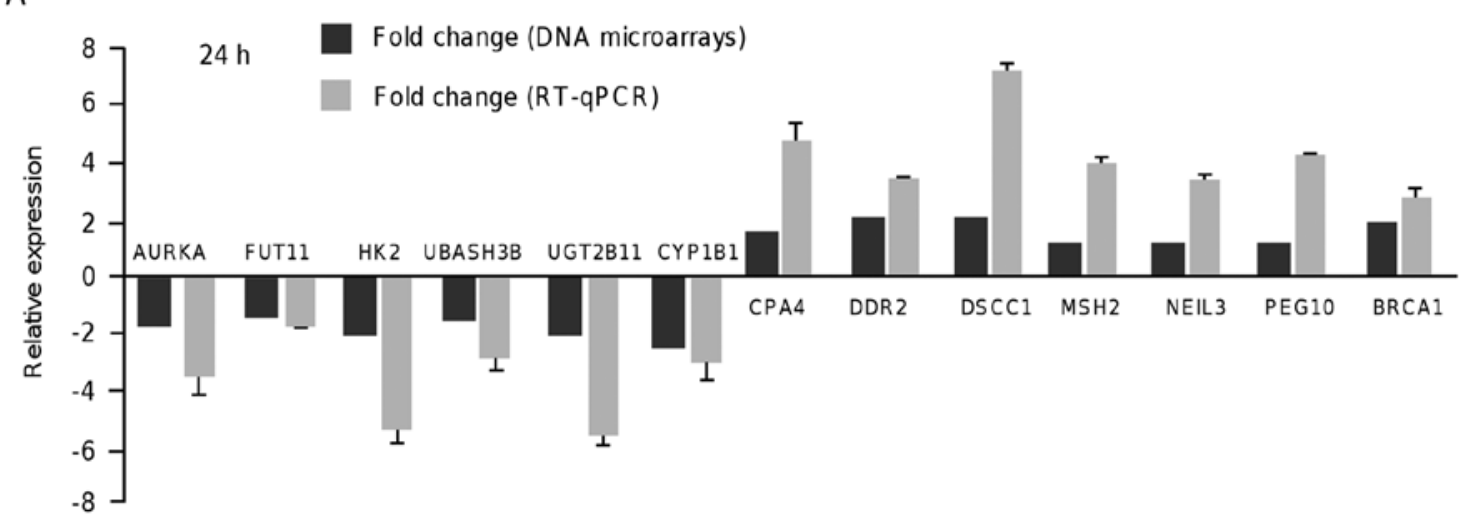

B

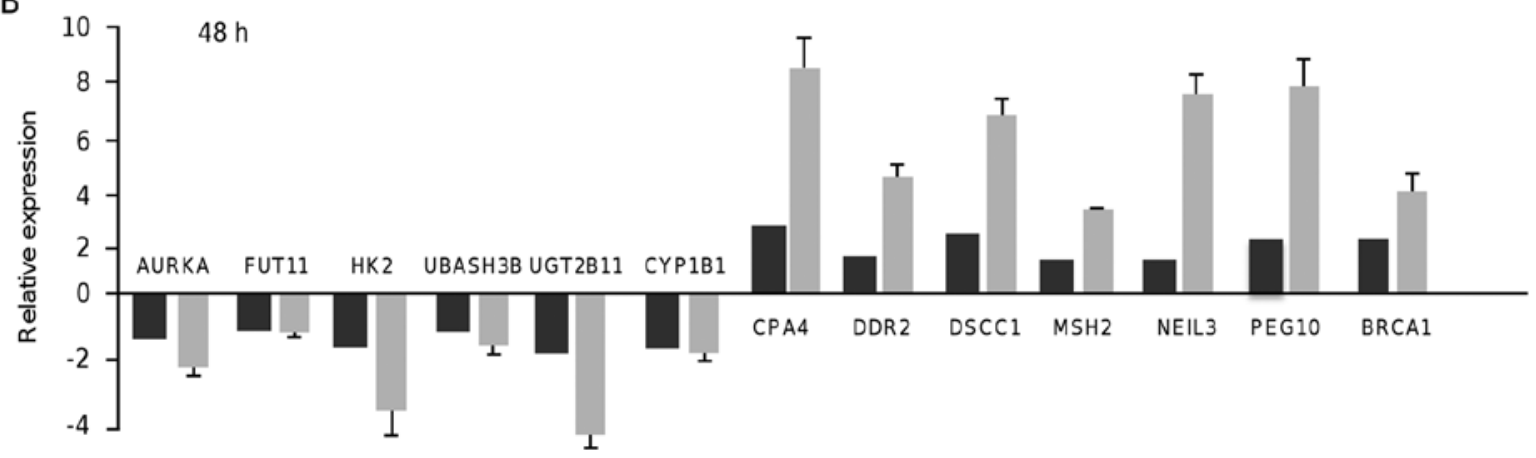

Figure 2. Validation of the DNA microarray data. RT-qPCR validation of 13 transcripts identified as differentially expressed by microarray hybridization in MDA-MB-231 breast cancer cells treated with resveratrol $(100 \mu \mathrm{M})$ at $24(\mathrm{~A})$ and $48 \mathrm{~h}(\mathrm{~B})$.

repair genes involved in homologous recombinational repair (RAD54L, EXO1, RECQL, BRCA1, BRCA2, BLM, DNA2, RBBP8, POLN), mismatch repair (MLH1, MSH2), Fanconi anemia pathway (FANCB, FANCD2, FANCL), base excision repair (UNG), DNA double-strand break repair (BRIP1, FAM175A) and repair of DNA inter-strand crosslinks (EME1) were upregulated at $48 \mathrm{~h}$ (Table III). Moreover, several genes involved in cell death, such as TP53I3, CCNE1 and SESN3, were overexpressed at $48 \mathrm{~h}$. Remarkably, 22 and 63 genes involved in the regulation of cell cycle progression were modulated at 24 and $48 \mathrm{~h}$, respectively (Tables II and III). Repressed cell cycle genes at $24 \mathrm{~h}$ included AURKA, PLK1, TXNIP, CCNB1, FAM83D, CDCA8, HJURP, CENPA, DLGAP5, BUB1, ASPM, and WEE1; whereas at $48 \mathrm{~h}$ of treatment, AURKA, PLK1, JUN, ARHGAP18, KIF20A, SH3KBP, EDN1 and PSMD11 were suppressed. To validate the DNA microarray data, we analyzed using RT-qPCR the expression of 13 modulated genes by resveratrol including six oncogenes at 24 and $48 \mathrm{~h}$. In all cases, the RT-qPCR data were similar to those obtained by the DNA microarray analyses (Fig. 2).

Resveratrol inhibits the G1/S phase transition in breast cancer cells. In our genome-wide analysis of gene expression in MDA-MB-231 cells treated with resveratrol at 24 and $48 \mathrm{~h}$, we identified 63 modulated genes involved in the cell cycle (Tables II and III). Thus, we decided to confirm the effects of resveratrol $(100 \mu \mathrm{M})$ on cell cycle progression in the MDA-MB-231 and MCF-7 breast cancer cell lines. The cells were treated with resveratrol and the distribution of cell cycle phases was determined by flow cytometric analysis as described in Materials and methods. The results showed that the percentage of cells in the G0-G1 phase increased from 56.7 to $77.3 \%$ at $24 \mathrm{~h}$ after resveratrol treatment, but retained a normal level at $48 \mathrm{~h}(54.7 \%)$ in comparison to the control (ethanol vehicle) in the MDA-MB-231 cells. This change was accompanied by an increment in the proportion of cells in the $\mathrm{S}$ phase from 26.4 in the control to $45.3 \%$ in the resveratrol-treated cells at $48 \mathrm{~h}$, but not at $24 \mathrm{~h}$ (Fig. 3A). However, we did not observed arrest in the G2-M phase in cells treated with resveratrol at 24 and $48 \mathrm{~h}$, in comparison with the control assays using nocodazole a known blocker of G2-M phase transition. In contrast, in the MCF-7 cells the percentage of cells in the G0-G1 phase did not significantly change after $24 \mathrm{~h}$ of resveratrol treatment in comparison with the control, whereas at $48 \mathrm{~h}$ we observed a slight but significant increase in cells in the G0-G1 phase (Fig. 3B). Taken altogether, these data indicate that resveratrol inhibits cell cycle progression and that its effects are cell line-specific.

Resveratrol targets the cell cycle regulators AURKA, PLK1, and BRCA1. Our DNA microarrays and RT-qPCR analysis indicated that the activators of cell cycle AURKA, CCND1 and CCNB1 were significantly repressed by resveratrol at 24 and $48 \mathrm{~h}$, whereas PLK1 was only suppressed at $24 \mathrm{~h}$. Moreover, the cell cycle repressor and AURKA/PLK1 inhibitor, BRCA1, was overexpressed at 24 and $48 \mathrm{~h}$ after resveratrol treatment. Thus, in order to confirm whether the alterations in mRNA abundance of these cell cycle genes also occur at the protein level, western blot assays using specific antibodies were performed. Results of the immunoblot analysis indicated that changes in 
A

MDA-MB-231
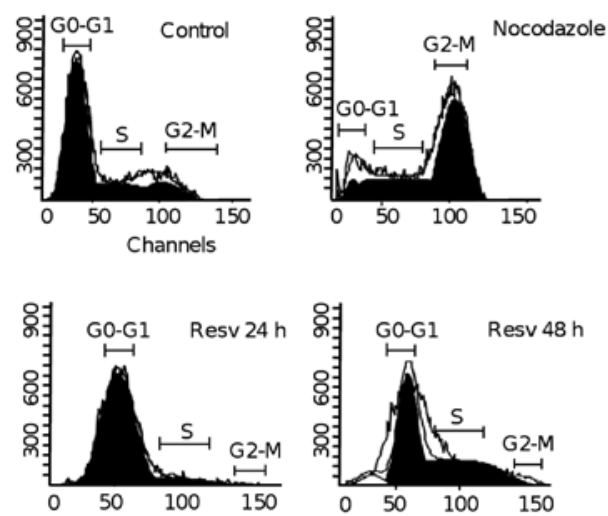

B
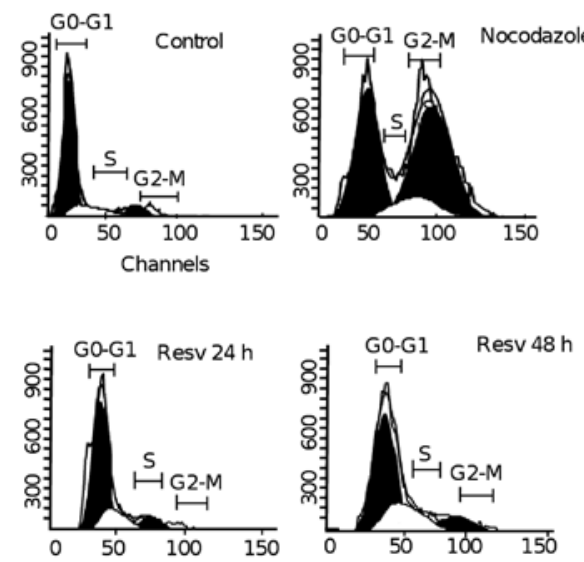

Cell cycle distribution

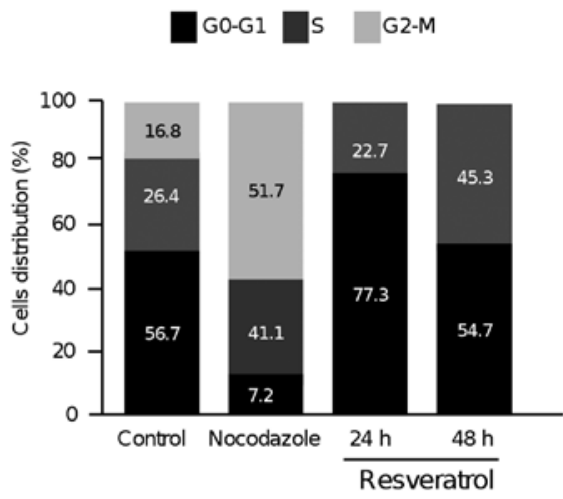

Cell cycle distribution

GO-G1 DS G2-M

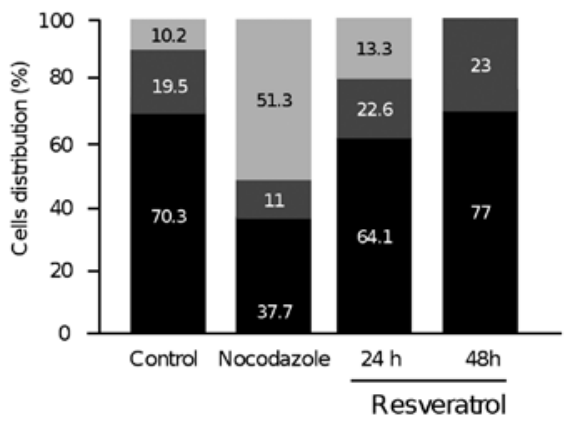

Figure 3. Resveratrol induces cell cycle arrest at the G0/G1 and S phases in breast cancer cells. Cell cycle analysis of (A) MDA-MB-231 and (B) MCF-7 cells treated with resveratrol $(100 \mu \mathrm{M})$, or ethanol vehicle and nocodazole as controls, for 24 and $48 \mathrm{~h}$. Percentage of cell distribution into cell cycle phases is shown in the right panel for each cell line. Resv, resveratrol.

the protein levels of these cell cycle genes were correlated with changes at the mRNA level. Data showed that after $24 \mathrm{~h}$ of resveratrol treatment, AURKA levels were markedly decreased (95\%) in both the MDA-MB-231 and MCF-7 cells in comparison with the control (Fig. 4A and B). In contrast, no significant changes were detected at $48 \mathrm{~h}$ in both cell lines. In addition, PLK1 was slightly but significantly decreased by resveratrol at 24 and $48 \mathrm{~h}$ in the MDA-MB-231 cells, but not in the MCF-7 cells (Fig. 4C and D). On the other hand, BRCA1 protein levels increased at $24 \mathrm{~h}$ whereas no significant changes were identified after $48 \mathrm{~h}$ of resveratrol treatment in the MDA-MB-231 cells (Fig. 4A). In addition, we confirmed the suppression of CCND1 and CCNB1 cyclins, which were previously described as two cell cycle markers modulated by resveratrol in MCF-7 and MDA-MB-231 cells (21).

\section{Discussion}

Here, we identified novel targets of resveratrol that may represent potential therapeutic targets in breast cancer. Our data showed that, after 24 and $48 \mathrm{~h}$ of resveratrol treatment, key genes involved in the regulation of cell cycle progression and DNA repair processes were modulated. These data are relevant as the development of breast cancer is frequently associated with disorders in the regulation of the cell cycle, and enhanced DNA repair is linked to chemotherapy resistance of tumor cells. Failure of the quality control checkpoints or a loss of balance of regulatory molecules plays a major role in carcinogenesis. Of the many regulatory cell cycle checkpoints, the acquisition of abnormalities at the G1/S checkpoint appears to be the most crucial step in the genesis and progression of cancer. Here, we found that resveratrol inhibited the proliferation of MDA-MB-231 and MCF-7 cells by blocking G0-G1 phase transition at $24 \mathrm{~h}$. In agreement with our data, several reports indicate that resveratrol activates apoptosis and inhibits cell proliferation of tumor cells $(22,23)$. Remarkably, we identified two novel targets of resveratrol, the AURKA and PLK1 kinases, which represent pivotal proteins related to cell division. Previous studies indicate that overexpression of AURKA and PLK1 promote cell proliferation through activation of G1/S cell cycle transition in multiple types of carcinomas (24). AURKA belongs to a small family of serine/treonine kinases with evolutionarily conserved structure and participates in mitosis. AURKA is upregulated in a wide range of malignancies and it acts as an oncogene both in vitro and in vivo, by enhancing G1-S transition, protecting cells from apoptosis, inducing centrosome amplification, promoting multipolar spindle formation and genomic instability, and conferring 
A

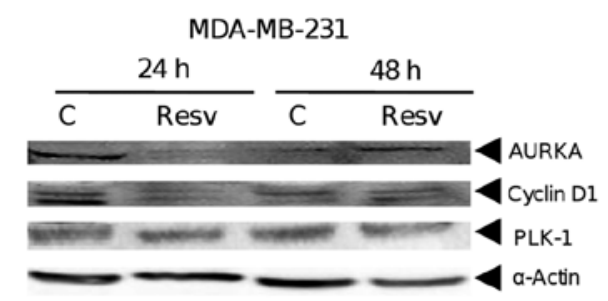

C
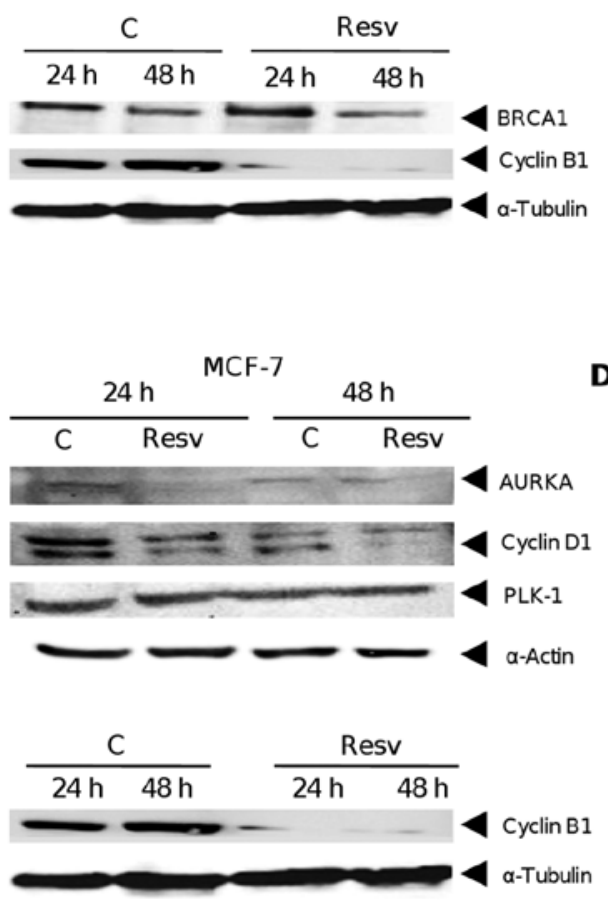

B
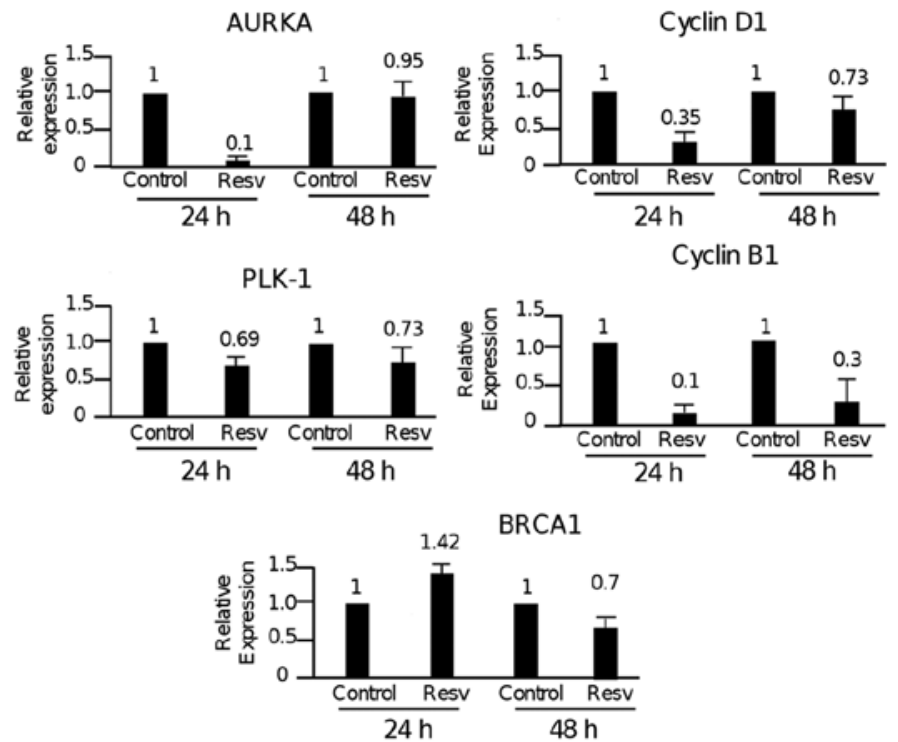

AURKA
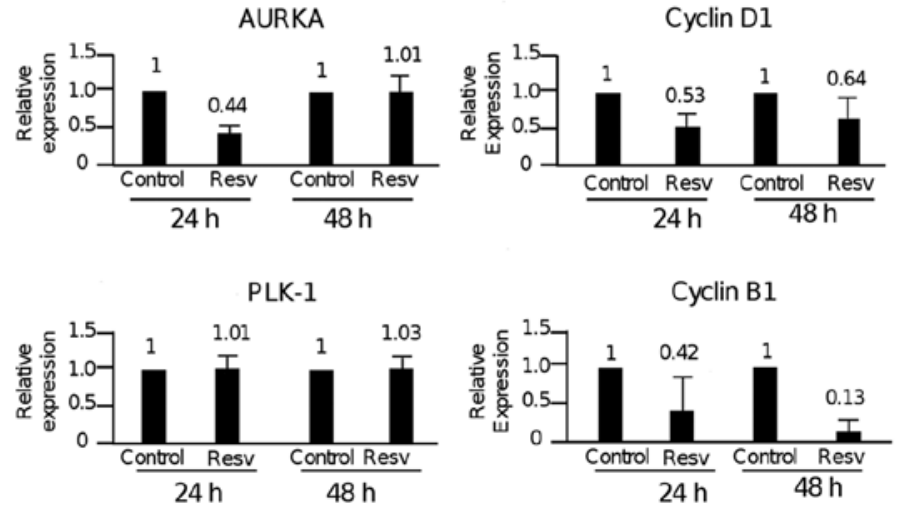

Figure 4. Resveratrol targets cell cycle proteins. Western blot analysis of (A) MDA-MB-231 and (C) MCF-7 cells treated with resveratrol (100 $\mu$ M, Resv), or non-treated (control) during 24 and $48 \mathrm{~h}$, using AURKA (1:1,000), cyclin D1 (1:1,000); PLK1 (1:500), BRCA1 (1:1,000) and cyclin B (1:1,000) antibodies. Actin $(1: 1,000)$ and tubulin $(1: 1,000)$ antibodies were used as control. (B and D) Densitometric analysis of bands in panels A and C. Data were normalized using actin or tubulin expression. Images are representative of three independent experiments. Resv, resveratrol.

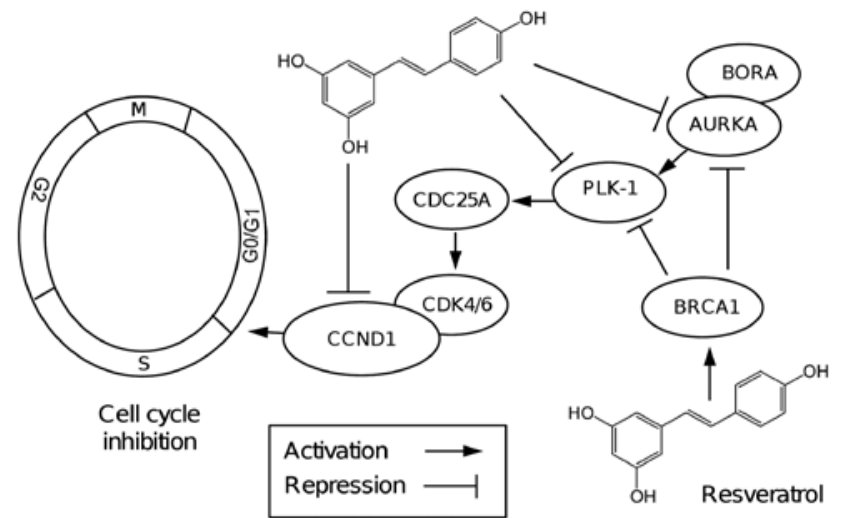

Figure 5. Model for the cell cycle regulation by resveratrol in MDA-MB-231 cells. PLK1 promotes the recruitment of AURKA which can self-activate. AURKA in complex with BORA activates PLK1. Phosphorylated PLK1 can activate the cell division cycle 25 (CDC25) to remove inhibitory phosphate from the CDK4/6 complex to facilitate G0/G1 to S phase transition. BRCA1 inhibits the PLK1 kinase activity by modulating its interaction with AURKA and BORA. Our data indicate that resveratrol reduces mRNA and protein levels of CCND1, AURKA and PLK1; and increases those of BRCA1. Resveratrol may directly inhibit cyclin D1 leading to an early cell cycle arrest by blocking G0/G1 phase transition. In addition resveratrol inhibits AURKA and PLK1 expression leading to an inactivation of $\mathrm{CDC} 25$ and $\mathrm{CCND} 1 / \mathrm{CDK} 4 / 6$ axis. resistance to antitumor agents. PLK1 is a checkpoint protein that participates in the regulation of centrosome maturation and spindle assembly, the removal of cohesins from chromosome arms, the inactivation of anaphase-promoting complex/cyclosome (APC/C) inhibitors, and the mitotic exit and cytokinesis. As PLK1 activates cyclin B and stimulates cell proliferation, its silencing results in an inactivation of cyclin $\mathrm{B} / \mathrm{Cdc} 2$ complex-mediated mitotic arrest followed by apoptosis (25). As these kinases may co-regulate several processes in mitosis, some overlap in the clinical performance of drugs that target PLK1 or aurora kinases is possible. However, the knowledge of the molecular factors that influence sensitivity and resistance to AURKA and PLK1 inhibitors remains limited.

On the other hand, we found that resveratrol $(100 \mu \mathrm{M})$ treatment resulted in the upregulation of the BRCA1 gene in MDA-MB-231 cells at 24 and $48 \mathrm{~h}$. Similar results were reported in breast cancer cell lines at low resveratrol doses $(30 \mu \mathrm{M})$ in previous research (26). BRCA1 is a tumor suppressor that plays critical roles in DNA repair, cell cycle checkpoint control, and maintenance of genomic stability. It also suppresses the cell cycle progression. Remarkably, a molecular interplay between AURKA, PLK1 and BRCA1 
proteins has been reported. BRCA1 inhibits the kinase activity of PLK1 by modulating the interactions of AURKA with hBora, and PLK1 (27). Our data confirm that resveratrol suppresses the expression of both CCND1 (G1-S transition) and CCNB1 (G2-M transition) cyclins at $24 \mathrm{~h}$ and $48 \mathrm{~h}$ in MDA-MB-231 and MCF-7 cells. Similar results for CCND1 and CCNB1 were previously described in SW-480 colorectal cancer cells and MCF-7 breast cancer cells (28). Taken all together, we propose a working model for cell cycle regulation by resveratrol in MDA-MB-231 cells (Fig. 5). In conclusion, the present study shows for the first time that resveratrol reduced mRNA and protein expression levels of AURKA and PLK1 two potential therapeutic targets in cancer. Our findings revealed the role of resveratrol in important cellular processes and in the modulation of key cell cycle regulators, which highlights its potential use as an adjuvant in breast cancer therapy.

\section{Acknowledgements}

CONACyT SALUD grants (233370 and 222335) supported this work. R.M. was supported by a CONACYT doctoral fellowship.

\section{References}

1. Le Corre L, Chalabi N, Delort L, Bignon YJ and Bernard-Gallon DJ: Resveratrol and breast cancer chemoprevention: Molecular mechanisms. Mol Nutr Food Res 49: 462-471, 2005.

2. Ferlay J, Soerjomataram I, Dikshit R, Eser S, Mathers C, Rebelo M, Parkin DM, Forman D and Bray F: Cancer incidence and mortality worldwide: Sources, methods and major patterns in GLOBOCAN 2012. Int J Cancer 136: E359-E386, 2015.

3. Wang Y, Wang Z, Qi Z, Yin S, Zhang N, Liu Y, Liu M, Meng J, Zang $\mathrm{R}$, Zhang $\mathrm{Z}$, et al: The negative interplay between Aurora A/B and BRCA1/2 controls cancer cell growth and tumorigenesis via distinct regulation of cell cycle progression, cytokinesis, and tetraploidy. Mol Cancer 13: 94, 2014.

4. McInnes C and Wyatt MD: PLK1 as an oncology target: Current status and future potential. Drug Discov Today 16: 619-625, 2011.

5. Sun JM, Yang LN, Xu H, Chang B, Wang HY and Yang G: Inhibition of Aurora A promotes chemosensitivity via inducing cell cycle arrest and apoptosis in cervical cancer cells. Am J Cancer Res 5: 1133-1145, 2015.

6. Vundru SS, Kale RK and Singh RP: $\beta$-Sitosterol induces G1 arrest and causes depolarization of mitochondrial membrane potential in breast carcinoma MDA-MB-231 cells. BMC Complement Altern Med 13: 280, 2013.

7. Singh CK, George J and Ahmad N: Resveratrol-based combinatorial strategies for cancer management. Ann NY Acad Sci 1290: 113-121, 2013.

8. Yuan L, Zhang Y, Xia J, Liu B, Zhang Q, Liu J, Luo L, Peng Z, Song $\mathrm{Z}$ and $\mathrm{Zhu} \mathrm{R}$ : Resveratrol induces cell cycle arrest via a p53-independent pathway in A549 cells. Mol Med Rep 11: 2459-2464, 2015.

9. Díaz-Chávez J, Fonseca-Sánchez MA, Arechaga-Ocampo E, Flores-Pérez A, Palacios-Rodríguez Y, Domínguez-Gómez G, Marchat LA, Fuentes-Mera L, Mendoza-Hernández G, Gariglio $\mathrm{P}$, et al: Proteomic profiling reveals that resveratrol inhibits HSP27 expression and sensitizes breast cancer cells to doxorubicin therapy. PLoS One 8: e64378, 2013.

10. Su D, Cheng Y, Liu M, Liu D, Cui H, Zhang B, Zhou S, Yang T and Mei Q: Comparision of piceid and resveratrol in antioxidation and antiproliferation activities in vitro. PLoS One 8: e54505, 2013.
11. Livak KJ and Schmittgen TD: Analysis of relative gene expression data using real-time quantitative PCR and the 2(-delta delta C(T)) method. Methods 25: 402-408, 2001.

12. In K, Park $\mathbf{J}$ and Park $H$ : Resveratrol at high doses acts as an apoptotic inducer in endothelial cells. Cancer Res Treat 38: 48-53, 2006.

13. Vanamala J, Reddivari L, Radhakrishnan S and Tarver C: Resveratrol suppresses IGF-1 induced human colon cancer cell proliferation and elevates apoptosis via suppression of IGF-1R/Wnt and activation of p53 signaling pathways. BMC Cancer 10: 238, 2010.

14. Alkhalaf M, El-Mowafy A, Renno W, Rachid O, Ali A and Al-Attyiah R: Resveratrol-induced apoptosis in human breast cancer cells is mediated primarily through the caspase-3-dependent pathway. Arch Med Res 39: 162-168, 2008.

15. Pandey PR, Okuda H, Watabe M, Pai SK, Liu W, Kobayashi A, Xing F, Fukuda K, Hirota S, Sugai T, et al: Resveratrol suppresses growth of cancer stem-like cells by inhibiting fatty acid synthase. Breast Cancer Res Treat 130: 387-398, 2011.

16. Liu B, Zhou Z, Zhou W, Liu J, Zhang Q, Xia J, Liu J, Chen N, Li $\mathrm{M}$ and Zhu R: Resveratrol inhibits proliferation in human colorectal carcinoma cells by inducing G1/S phase cell cycle arrest and apoptosis through caspase/cyclin CDK pathways. Mol Med Rep 10: 1697-1702, 2014.

17. García-Zepeda SP, García-Villa E, Díaz-Chávez J, Hernández-Pando R and Gariglio P: Resveratrol induces cell death in cervical cancer cells through apoptosis and autophagy. Eur J Cancer Prev 22: 577-584, 2013.

18. Chang I, Mitsui Y, Fukuhara S, Gill A, Wong DK, Yamamura S, Shahryari V, Tabatabai ZL, Dahiya R, Shin DM, et al: Loss of miR-200c up-regulates CYP1B1 and confers docetaxel resistance in renal cell carcinoma. Oncotarget 6: 7774-7787, 2015.

19. Pan B, Zheng S, Liu C and Xu Y: Suppression of IGHG1 gene expression by siRNA leads to growth inhibition and apoptosis induction in human prostate cancer cell. Mol Biol Rep 40: 27-33, 2013.

20. Chen Y, Li D and Li S: The Alox5 gene is a novel therapeutic target in cancer stem cells of chronic myeloid leukemia. Cell Cycle 8: 3488-3492, 2009.

21. Pozo-Guisado E, Alvarez-Barrientos A, Mulero-Navarro S, Santiago-Josefat B and Fernandez-Salguero PM: The antiproliferative activity of resveratrol results in apoptosis in $\mathrm{MCF}-7$ but not in MDA-MB-231 human breast cancer cells: Cell-specific alteration of the cell cycle. Biochem Pharmacol 64: 1375-1386, 2002.

22. Meeran SM and Katiyar SK: Cell cycle control as a basis for cancer chemoprevention through dietary agents. Front Biosci 13: 2191-2202, 2008.

23. León-Galicia I, Díaz-Chávez J, García-Villa E, Uribe-Figueroa L, Hidalgo-Miranda A, Herrera LA, Alvarez-Rios E, Garcia-Mena J and Gariglio P: Resveratrol induces downregulation of DNA repair genes in MCF-7 human breast cancer cells. Eur J Cancer Prev 22: 11-20, 2013.

24. Lee SY, Jang C and Lee KA: Polo-like kinases (plks), a key regulator of cell cycle and new potential target for cancer therapy. Dev Reprod 18: 65-71, 2014.

25. Li Y, Zhang DY, Ren Q, Ye F, Zhao X, Daniels G, Wu X, Dynlacht $B$ and Lee P: Regulation of a novel androgen receptor target gene, the cyclin B1 gene, through androgen-dependent E2F family member switching. Mol Cell Biol 32: 2454-2466, 2012.

26. Fustier P, Le Corre L, Chalabi N, Vissac-Sabatier C, Communal Y, Bignon YJ and Bernard-Gallon DJ: Resveratrol increases BRCA1 and BRCA2 mRNA expression in breast tumour cell lines. Br J Cancer 89: 168-172, 2003.

27. Zou J, Rezvani K, Wang H, Lee KS and Zhang D: BRCA1 downregulates the kinase activity of Polo-like kinase 1 in response to replication stress. Cell Cycle 12: 2255-2265, 2013.

28. Joe AK, Liu H, Suzui M, Vural ME, Xiao D and Weinstein IB: Resveratrol induces growth inhibition, S-phase arrest, apoptosis, and changes in biomarker expression in several human cancer cell lines. Clin Cancer Res 8: 893-903, 2002. 